\title{
Eating patterns and mental health problems in early adolescence - a cross-sectional study of 12-13-year-old Norwegian schoolchildren
}

\author{
Inger M Oellingrath ${ }^{1, *}$, Martin V Svendsen ${ }^{2}$ and Ingebjørg Hestetun ${ }^{3}$ \\ ${ }^{1}$ Faculty of Health and Social Sciences, Department of Health Studies, Telemark University College, PO Box \\ 201, 3914 Porsgrunn, Norway: ${ }^{2}$ Department of Occupational and Environmental Medicine, Telemark Hospital, \\ Skien, Norway: ${ }^{3}$ Department of Child and Adolescent Psychiatry, Telemark Hospital, Skien, Norway
}

Submitted 14 January 2013: Final revision received 21 August 2013: Accepted 28 August 2013: First published online 100 0ctober 2013

\begin{abstract}
Objective: To investigate the association between eating patterns and mental health problems in young Norwegian adolescents (12-13 years of age).

Design: Cross-sectional study. Dietary information was reported by parents using a retrospective FFQ. Eating patterns were identified using principal component analysis. The Strengths and Difficulties Questionnaire was used to measure mental health problems. The association between eating patterns and mental health problems was examined using multiple logistic regression analysis.

Setting: Primary schools, Telemark County, Norway.

Subjects: Children ( $n$ 1095) aged 12-13 years and their parents.

Results: Children with high scores on a 'varied Norwegian' eating pattern were less likely to have indications of any psychiatric disorders (adjusted OR $=0.5 ; 95 \% \mathrm{CI}$ $0 \cdot 3,1 \cdot 0$ ) and hyperactivity-inattention disorders (adjusted OR $=0 \cdot 4 ; 95 \% \mathrm{CI} 0 \cdot 2,0 \cdot 8$ ) than children with low scores on this pattern. Children with high scores on a 'junk/ convenient' eating pattern were more likely to have indications of hyperactivityinattention disorders (adjusted OR $=3 \cdot 4 ; 95 \%$ CI 1.3, 8.6) than children with low scores on this pattern. Children with high scores on a 'snacking' eating pattern were more likely to have indications of conduct/oppositional disorders (adjusted $\mathrm{OR}=3 \cdot 8 ; 95 \%$ CI $1 \cdot 2,11 \cdot 5)$ than those with low scores on this eating pattern. Conclusions: We identified a significant association between eating patterns and mental health problems in young adolescents, independently of physical activity, sedentary activity and background variables. A diverse diet rich in unrefined plant foods, fish and regular meals was associated with better mental health, while energy-dense, nutrient-poor diets and irregular meals were associated with poorer mental health.
\end{abstract}

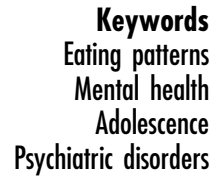

Mental health problems among adolescents represent a growing global health problem ${ }^{(1)}$. Common mental disorders in adults often first emerge in childhood and adolescence $^{(2)}$. As many as half of lifetime psychiatric disorders will emerge in early adolescence before the age of 14 years $^{(3)}$. Norwegian health data indicate that $15-20 \%$ of children and adolescents have reduced function due to mental disorder symptoms and that $8 \%$ have severe symptoms that meet the requirements for a psychiatric diagnosis $^{(4)}$. Mental health problems are more commonly seen among adolescents than among younger children $^{(4)}$.

In addition, unhealthy dietary habits, such as high intakes of added sugar and saturated fat, low intakes of fruit and vegetables, and irregular breakfast eating, have been reported among Norwegian adolescents ${ }^{(5,6)}$.
Adequate nutrition is known to be an important factor in the development of the brain and for cognitive function during childhood and adolescence ${ }^{(7,8)}$. Furthermore, a growing number of studies, including three Norwegian publications, have suggested a cross-sectional association between unhealthy eating habits and mental health problems in children and adolescents ${ }^{(9-20)}$. In the Norwegian studies, breakfast skipping and high consumption of sugar-containing soft drinks and sweets were associated with various mental health problems ${ }^{(9,12,13)}$. In one newly published Australian prospective study, healthy diets and improvements in diet quality were associated with improvements in mental health in adolescence ${ }^{(15)}$. The latter highlights the importance of healthy eating in adolescence and the potential role of diet in preventing mental health problems throughout life ${ }^{(15)}$. 
Previous research on associations between children's diet and health has focused mainly on isolated nutrients, single meals or certain food groups. Recently, the use of dietary patterns has become popular in studies of children's diet. Dietary patterns provide an overview of diet and facilitate exploration of overall diet and diet-disease associations that are not revealed when single nutrients or food items are considered in isolation ${ }^{(21,22)}$. Results from studies of dietary patterns may more easily be translated into diet recommendations and public health interventions ${ }^{(23)}$. The most commonly used method for detecting dietary patterns is principal component analysis (PCA). The dietary pattern approach has rarely been used to examine associations between diet quality and mental health problems among young people, and further studies are recommended ${ }^{(23)}$.

A few studies documenting a significant relationship between overall dietary patterns and mental health problems in adolescent populations have been published in recent years ${ }^{(10,16,18)}$. In the Western Australian Pregnancy Cohort (Raine) Study, cross-sectional associations between a 'Western-style' dietary pattern (takeaway foods, confectionery and red meat), poor externalizing and internalizing behaviour, and diagnosis of attentiondeficit hyperactivity disorder (ADHD) were observed at age 14 years $^{(16,18)}$. A newly published Chinese study observed a relationship between an unhealthy dietary pattern and emotional symptoms in 11-16-year-olds ${ }^{(10)}$. Given the limited number of studies completed in a limited number of countries, more are needed to build knowledge in the field. No studies examining the association between overall eating patterns and mental health problems have been conducted among Norwegian adolescents.

We have previously reported four distinct PCA-derived eating patterns in young Norwegian adolescents (12-13 years of age $)^{(24)}$. Parent-reported mental health problems were collected simultaneously using the Strengths and Difficulties Questionnaire (SDQ) and impact supplement ${ }^{(25)}$. The aim of the present study was to investigate the association between eating patterns and mental health problems among young Norwegian adolescents.

\section{Methods}

\section{Participants and study design}

The present data were obtained from a cross-sectional study of diet, physical activity and BMI development in primary-school children in Telemark County, Norway. The data were collected in the spring of 2010, from children in primary school grade 7 (age 12-13 years). The detailed data collection methods have been described previously $^{(24,26)}$. In brief, all 104 primary schools in Telemark County were invited to participate in the study, and fifty-three agreed. Written parental consent to inclusion in the study was received for 1095 out of 1503 invited children (73\%), representing about half of the county's grade 7 pupils.

The study was conducted in accordance with the guidelines laid down in the Declaration of Helsinki and the research protocol was approved by the the Regional Committee for Ethics in Medical Research and the Norwegian Data Inspectorate.

\section{Dietary information}

The children's food and drink intake was reported by their parents using a retrospective FFQ, which asked about habitual daily consumption of forty food items, eleven types of drink, thirteen types of snacks (between meals) and five main meals (breakfast, lunch, afternoon meal, dinner, supper) during the last 6 months. This questionnaire was based on a short validated FFQ developed for use with children in grades 4 and 8 in Norway ${ }^{(27)}$, but was modified to include more dietary questions. Before the present study, the FFQ was tested on a sample of parents and followed up by qualitative interviews ${ }^{(28)}$. Response alternatives and other details have been reported previously ${ }^{(24,26)}$. As we used meal and snacking events in addition to food consumption frequencies as input variables in the PCA, the components were denoted 'eating patterns' rather than 'dietary patterns'.

\section{Mental bealtb problems}

The adolescents' mental health problems were measured using the parental version of the SDQ (P4-16) ${ }^{(25)}$. The instrument includes a twenty-five-item informant-rated SDQ and an impact supplement that asks whether the parents think that the young person has a problem. If so, further information is given about chronicity, distress, social impairment and burden for others ${ }^{(25)}$.

Each of the twenty-five items is scored on a 3-point Likert-type scale from 0 to 2 . Four subscales comprising five items each cover emotional symptoms, conduct problems, hyperactivity and peer problems. These are summed to give a total difficulties score. A fifth subscale comprising five items covers pro-social behaviour. Information from the first three SDQ subscales (emotional symptoms, conduct problems, hyperactivity) and the SDQ impact supplement were combined using an algorithm defined by Goodman et al. ${ }^{(29)}$.

The algorithm makes separate predictions for indications of emotional disorders (anxiety-depressive disorders), conduct disorders (conduct/oppositional disorders) and hyperactivity disorders (hyperactivity-inattention disorders, ADHD) ${ }^{(29)}$. These three scales are further combined to generate an overall prediction of the indication of a psychiatric disorder. The algorithm predicts a psychiatric disorder as being 'likely' (high risk), 'possible' (borderline) or 'unlikely' (normal). As Norwegian norms are not established, British norms were used to predict the categories ${ }^{(30)}$. In the present study, we combined the 'possible' and 'likely' category into 'possible/likely', and all comparisons were 
made between this category and the category 'unlikely'. The algorithm allows scale scores to be pro-rated if at least three of the five scale items on the subscales are complete. Normally, the algorithm requires there to be at least two independent informants to meet the criteria for 'likely' on the hyperactivity-inattention scale. In the present study, the information was reported by parents only. We therefore made an adjustment to allow the criteria for the category 'possible/likely' to be met if there was just one informant, provided that the informant produced a score within the defined 'possible/likely' category range.

In the present study, we use 'mental health problems' as an overall term to denominate the indications of psychiatric disorders explained by the algorithm. We did not include the peer problem and pro-social behaviour subscales in the analysis, as they were considered as less relevant to the study aim.

\section{Socio-economic variables and family structure}

In addition to providing dietary information and mental health data, the parents reported their educational level, family income and family structure.

Parental educational level was divided into three categories: 'primary and lower secondary education' (10 years or less), 'upper secondary education' (3 to 4 years) and 'university or university college'.

Family income was divided into three categories: 'both parents $<$ NOK 300000 ', 'one parent $\geq$ NOK 300000 ' and 'both parents $\geq$ NOK 300000 ' (where NOK is Norwegian kroner and NOK $300000=€ 40849$ as at 12 December 2012).

Family structure was divided into four categories: 'child living with both parents', 'child living with one parent', 'child living with one parent and stepfather/stepmother' and 'child living equal amounts with mother and father in separate homes'.

\section{Physical activity and sedentary activity}

A variable categorizing physical leisure activity by reference to other children was used as an indicator of the children's physical activity level. Parents indicated on a scale from 1 to 5 whether the child was 'less physically active than other children of the same age' or 'more physically active than other children of the same age'. The question was taken from a battery of validated questions used in a study of children's activity and inactivity in the Netherlands ${ }^{(31)}$ and translated into Norwegian for use in the present study. Parent-reported sedentary activity was defined as time spent on screen-based activities and other sedentary activities outside school. These activities were combined and divided into two categories: ' $<4 \mathrm{~h} / \mathrm{d}$ ' and ' $\geq 4 \mathrm{~h} / \mathrm{d}$ '.

\section{BMI categories}

The weight and height of the children were measured by public health nurses at each school. The children were weighed wearing light clothing (i.e. trousers, T-shirt, socks) using calibrated, electronic scales measuring in
$100 \mathrm{~g}$ increments. BMI $\left(\mathrm{kg} / \mathrm{m}^{2}\right)$ of each child was calculated on the basis of these measurements. Child BMI categories were calculated using the International Obesity Task Force cut-off points (underweight, normal weight, overweight, obese), based on growth curves and BMI of 17,25 and $30 \mathrm{~kg} / \mathrm{m}^{2}$ at age 18 years ${ }^{(32,33)}$. The respective cut-off points for $12 \cdot 5$-year-old boys and girls were used. Due to small numbers, we included underweight children in the normal weight group and obese children in the overweight group.

\section{Statistical analysis}

Four distinct eating patterns had previously been identified by PCA of the reported dietary responses, representing both healthy and unhealthy eating behaviour ${ }^{(24)}$. The eating patterns were named after the nature of the foods, beverages and meals within each pattern, as follows: (i) a 'junk/convenient' pattern, characterized by high-energy processed fast foods, refined grains, cakes and sweets; (ii) a 'varied Norwegian' pattern, characterized by food items typical of a traditional Norwegian diet, including unrefined plant foods, fish, water and regular breakfast and lunch; (iii) a 'snacking' pattern, characterized by sugar-rich snack items and drinks, low intake of vegetables and brown bread, low frequency of eating breakfast and dinner and high frequency of eating between meals; and, finally, (iv) a 'dieting' pattern, containing foods and drinks often associated with dieting and weight control. Individuals were given factor scores for each of the eating patterns. Positive factor scores indicate high intake of foods, drinks and meals within the respective pattern, while negative factor scores indicate low intake. The factor scores for each eating pattern were ranked into categorical variables (tertiles).

We used multiple logistic regression analysis to examine the association between eating patterns and mental health problems, as independent and dependent variables, respectively. Adjusted odds ratios and 95\% confidence intervals were calculated for emotional, conduct, hyperactivity and any psychiatric disorders. The potential confounding variables in the logistic regression models were maternal and paternal education, family income, family structure, child physical activity, sedentary activity, BMI category and gender. We applied forward conditional selection, which included variables significantly associated with mental health problems in each model. Informants with missing values on a given variable were included as a separate category.

Only participants for whom complete data were available on each of the mental health problem scales and dietary patterns were used in the present analyses. For all tests, $P<0.05$ was considered significant. The questionnaires were scanned by Eyes and Hands (Readsoft Forms, Helsingborg, Sweden) and additional manual control routines were used throughout the process. All statistical analyses were carried out using the statistical software package SPSS Statistics version 17. 


\section{Results}

Complete data on parent-reported mental health problems and children's diet were obtained for 789 children ( $52 \%$ of the invited cohort). Characteristics of the sample are given in Table 1 .

In total, $9 \%$ of the 789 children had symptoms of an extent and severity that indicated that a psychiatric disorder was possible or likely (Table 2). The most frequently reported indications were of hyperactivity disorders and conduct disorders, while indications of emotional disorders were reported less frequently.

The adjusted multiple regression model for the association between eating patterns and mental health problems
(Table 3) showed that adolescents with high scores on the 'varied Norwegian' eating pattern were less likely to have indications of 'any psychiatric disorders' than those with lower scores on this eating pattern. Further, adherence to the 'varied Norwegian' eating pattern was associated with a lower likelihood of indications of hyperactivity disorders. Adolescents with moderate to high scores on the 'junk/ convenient' eating pattern were more likely to have indications of hyperactivity disorders than adolescents with low scores on this pattern. Furthermore, high scores on the 'snacking' eating pattern were associated with a higher likelihood of indications of conduct disorders. No significant associations were observed between the 'dieting' eating pattern and mental health problems.

Table 1 Characteristics of study participants: 12-13-year-old children and their parents $(n 789)^{\star}$, Telemark County, Norway, spring 2010

\begin{tabular}{|c|c|c|c|}
\hline Characteristic & Category & $n$ & $\%$ \\
\hline \multirow[t]{3}{*}{ Child's BMI category ${ }^{+}$} & Normal weight & 572 & 72 \\
\hline & Overweight & 110 & 14 \\
\hline & Missing & 108 & 14 \\
\hline \multirow{4}{*}{ Maternal education } & Primary/lower secondary & 91 & 12 \\
\hline & Upper secondary & 269 & 34 \\
\hline & University/university college & 388 & 49 \\
\hline & Missing & 41 & 5 \\
\hline \multirow[t]{4}{*}{ Paternal education } & Primary/lower secondary & 88 & 11 \\
\hline & Upper secondary & 343 & 44 \\
\hline & University/university college & 276 & 35 \\
\hline & Missing & 82 & 10 \\
\hline \multirow[t]{4}{*}{ Family income } & Both parents $<$ NOK 300000 & 86 & 11 \\
\hline & One parent $\geq$ NOK 300000 & 333 & 42 \\
\hline & Both parents $\geq$ NOK 300000 & 342 & 45 \\
\hline & Missing & 18 & 2 \\
\hline \multirow[t]{5}{*}{ Family structure, child living with: } & Both parents & 536 & 68 \\
\hline & One parent & 92 & 12 \\
\hline & One parent and stepfather/stepmother & 82 & 10 \\
\hline & Mother/father equally, separate homes & 75 & 10 \\
\hline & Missing & 4 & 1 \\
\hline \multirow[t]{2}{*}{ Child's gender } & Boy & 395 & 50 \\
\hline & Girl & 393 & 50 \\
\hline \multirow{4}{*}{ Child's physical activity } & Less than other children & 90 & 11 \\
\hline & Same as other children & 355 & 45 \\
\hline & More than other children & 330 & 42 \\
\hline & Missing & 14 & 2 \\
\hline \multirow[t]{3}{*}{ Inactivity of the child } & $\geq 4 \mathrm{~h} / \mathrm{d}$ & 413 & 52 \\
\hline & $<4 \mathrm{~h} / \mathrm{d}$ & 350 & 44 \\
\hline & Missing & 26 & 3 \\
\hline
\end{tabular}

*Sample with complete data on diet and mental health problems. Distribution of each background variable is given $(n 789)$. tInternational Obesity Task Force cut-off points for $12 \cdot 5$-year-old boys and girls ${ }^{(32,33)}$.

Table 2 Mental health problems of $12-13$-year-old children $(n 789)^{\star}$ according to the extended SDQ (SDQ symptom scores combined with impact scale scores), Telemark County, Norway, spring 2010

\begin{tabular}{|c|c|c|c|}
\hline Mental health problem & Category & $n$ & $\%$ \\
\hline \multirow[t]{2}{*}{ Indications of any psychiatric disorders } & Unlikely & 717 & 91 \\
\hline & Possible/likely & 72 & 9 \\
\hline \multirow[t]{2}{*}{ Indications of emotional disorders } & Unlikely & 773 & 98 \\
\hline & Possible/likely & 16 & 2 \\
\hline \multirow[t]{2}{*}{ Indications of conduct disorders } & Unlikely & 761 & 96 \\
\hline & Possible/likely & 28 & 4 \\
\hline \multirow[t]{2}{*}{ Indications of hyperactivity disorders } & Unlikely & 745 & 94 \\
\hline & Possible/likely & 44 & 6 \\
\hline
\end{tabular}

SDQ, Strengths and Difficulties Questionnaire.

${ }^{*}$ Sample with complete data on diet and mental health problems. Distribution of each mental health variable is given ( $n$ 789). 


\section{Discussion}

In the present study, we found a significant relationship between eating patterns and mental health problems in young adolescents, independently of physical activity, sedentary activity and various background factors. Adolescents with high adherence to the 'varied Norwegian' eating pattern (recommended nutrient-dense foods like fruits, vegetables, unrefined grains and fish combined with regular meals) were less likely to have indications of 'any psychiatric disorders' and a reduced likelihood of hyperactivity disorders. Moderate to high adherence to the 'junk/convenient' eating pattern (energy-dense and processed foods) was associated with indications of hyperactivity disorders. Adolescents with high adherence to a 'snacking' eating pattern (unhealthy snacking and irregular meals) were more likely to have indications of conduct disorders.

A few studies have previously investigated associations between diet and mental health problems in adolescents, and three studies have used overall dietary patterns in their analyses ${ }^{(10,16,18)}$. Different assessments of mental health problems and diet, as well as a different eating pattern composition, make direct comparison with our results difficult. However, some clear similarities can be observed.

Our findings are in line with previous studies in which healthy eating patterns or food combinations of fruit, vegetables and fish have been associated with better mental health in adolescents ${ }^{(9,10,15-18)}$. In the Australian Raine study, high scores on a 'healthy' dietary pattern and high intake of fruits and vegetables were associated with a lower likelihood of ADHD diagnosis and better behaviour (lower symptoms of conduct problems) at age 14 years $^{(16,18)}$. Similar associations between a moderate intake of fruits and fish and a lower likelihood of behavioural problems were recently reported in a cohort study of Norwegian adolescents ${ }^{(9)}$.

The association observed between the unhealthy 'junk/convenient' eating pattern and indications of mental health problems is consistent with the Raine study, in which adolescents with high scores on a 'Western' dietary pattern were more likely to have an ADHD diagnosis ${ }^{(16)}$. The food composition of the Australian 'Western' dietary pattern had several similarities with the 'junk/convenient' eating pattern in the present study, as both represent energy-dense diets rich in junk foods and other processed foods, refined grains, cakes and sweets. Similar crosssectional associations between dietary patterns featuring energy-rich foods and junk food and indications of hyperactivity have previously been reported in younger children $^{(11,19)}$.

Our results further support previous findings from two Norwegian adolescent cohort studies in which high intakes of unhealthy foods like sugar-sweetened soft drinks, sweets and snacks were associated with behavioural problems ${ }^{(9,12)}$. 
This is also in line with the Raine study, in which the 'Western' dietary pattern (rich in confectionery and unhealthy snacks) was associated with poorer behaviour (symptoms of conduct problems) $^{(18)}$.

An association between diet and emotional problems in adolescence has been reported in a few previous studies $^{(10,15,17,18)}$. The absence of significant associations between the defined eating patterns and indications of emotional disorders (anxiety-depressive disorders) in the present study may be due to the relatively low number of adolescents with indications of emotional disorders. This increases the probability of not detecting true associations that are present (type II errors). The low rate of emotional disorders may be due to the relatively young age of the participants in our study, as emotional problems are known to increase during adolescence ${ }^{(2,3)}$. Further, the use of parents as the only informants may have resulted in some underestimation of emotional symptoms in our sample (30,34,35). $^{\text {. }}$

Possible explanations for associations between diet and mental health problems are complex, and have previously been reviewed in detail by others ${ }^{(7,23)}$. Nutrient composition and meal patterns can have immediate and long-term beneficial or adverse effects ${ }^{(7)}$. Unlike the nutrient-poor 'junk/convenient' and 'snacking' eating patterns, the 'varied Norwegian' eating pattern represents a diverse diet rich in unrefined plant foods and fish, i.e. foods providing several important nutrients such as folic acid, thiamin, vitamin $\mathrm{B}_{12}$, Se, $\mathrm{Zn}$ and $n-3$ fatty acids, which have previously been found to be associated with better behaviour and cognitive status among adolescents ${ }^{(23)}$. Furthermore, the "varied Norwegian' eating pattern includes regular breakfast and lunch. Previous studies have associated regular breakfast eating and breakfast quality with good mental health, well-being, better mood and better performance among adolescents $^{(7,13,20,36)}$. Meal regularity may also be an indicator of good family regularity and functioning, which has been linked to better mental health in adolescents ${ }^{(37,38)}$. A main purpose of the present study was to focus on overall eating behaviour using eating patterns rather than single components in the analyses. Although causal relationships cannot be firmly identified, the results of the present study indicate that a varied, nutrient-dense diet and regular meals are associated with good mental health in adolescents. The 'varied Norwegian' eating pattern was highly comparable to current Norwegian dietary advice.

It is important to recognize that observed associations between eating patterns and mental health problems may be bidirectional, as mental health problems may promote unhealthy eating. Symptoms of ADHD include inattention, impulsivity and hyperactivity, and may imply a higher risk of developing unhealthy eating patterns ${ }^{(39-42)}$. It is also likely that adolescents with conduct problems may be less inclined to respect family rules and more likely to skip family meals and engage in uncontrolled 'snacking' behaviour. Emotional problems have been linked to comfort eating ${ }^{(43)}$. However, a reverse causal relationship between diet and depression was not supported by a recent Australian adolescent prospective study $^{(15)}$. To further investigate possible longitudinal effects and to clarify the direction of the associations observed in our study, follow-up studies are needed.

Our study has strengths, but also limitations that should be recognized. Two strengths are the relatively large number of informants and the acceptable response rate. Furthermore, the study included several background and lifestyle variables considered important with regard to diet and mental health variability. A further important strength is the use of PCA-derived eating patterns as a measure of the children's overall dietary and meal habits, as opposed to individual nutrients, single food items and single meals ${ }^{(22)}$. The FFQ in the present study represented a wide range of commonly used food items, snack products, drinks and meals, resulting in robust factors (eating patterns) covering multiple items.

Another strength is the use of the extended SDQ, a brief instrument for psychiatric screening of children and adolescents that measures both positive attributes and symptoms of problems, as well as the impact of problems ${ }^{(25)}$. The extended SDQ is a valuable screening instrument for adolescent mental health problems when used in epidemiological research ${ }^{(44,45)}$. The SDQ has been widely used in studies of Norwegian community samples ${ }^{(46)}$. Very similar versions are available for parents, young people and teachers.

One limitation is the lack of cross-informant comparisons. The algorithm used in the study was developed and recommended for use with multiple informants. However, the method has also been used where parents are the only informants ${ }^{(47,48)}$. This involves limitations that should be recognized ${ }^{(30,49)}$. These limitations mainly concern potential underestimation of SDQ difficulty scores, which may in turn lead to some underestimation of mental health problems. Using British norms may have resulted in some underestimation of problems, as Norwegian studies generally report lower symptom scale means than found in Britain ${ }^{(35,46)}$. Prevalence estimation of mental health problems was not a main aim of our study.

The dietary reports may have been influenced by parental under-reporting of unhealthy items or over-reporting of healthy products, as parents will be less aware of what their children eat outside the home ${ }^{(50)}$. Further, biases caused by errors in parental memory cannot be excluded ${ }^{(51)}$. It is likely that the dietary data reflect the parents' 'dietary image' rather than the true habitual diet of the children ${ }^{(51)}$. However, the FFQ data were used to derive patterns which reflect dietary behaviour ${ }^{(52)}$. Dietary patterns are less likely to be distorted by misreporting than estimated intakes of energy, nutrients and food amounts. The reproducibility and validity of PCA-derived dietary patterns assessed using FFQ have previously been found to be comparable to those of patterns obtained using weighed dietary records ${ }^{(53-56)}$. 
Any parental misreporting of food items is likely to have attenuated the association between eating patterns and mental health problems.

It can be assumed that sociodemographic and lifestyle factors may have influenced the association between eating habits and mental health problems. Clear associations have previously been reported between eating patterns and gender, parental education levels, physical activity and the tracking of children's overweight ${ }^{(24,26)}$. Mental health problems in adolescents have been associated with several sociodemographic variables, physical activity and inactivity ${ }^{(14,44,57,58)}$. However, only minor changes were observed in the associations identified between eating patterns and mental health problems when adjusted for confounding variables, indicating an important association between the two. Nevertheless, adolescent mental health problems may be related to other biological and environmental factors not taken into account here.

One possible limitation on the results is bias due to non-responders. The participating parents had a somewhat higher educational level and total family income than the county's population in general. Further, we included only participants with complete data on diet and mental health variables in the analyses. However, because those with missing data on these variables did not differ substantially from the remaining informants with regard to background variables, we consider this problem to be limited. The missing data on BMI categories were mainly linked to the drop-out of two schools with organizational problems. Data collection was limited to one Norwegian county and the results are not necessarily representative of the national population.

The study's cross-sectional design eliminates the possibility of identifying causal relationships between children's eating patterns and mental health problems. To further investigate possible longitudinal effects and to clarify the direction of the associations, follow-up studies are needed. Hence, the results will form the baseline for further research, as we intend to repeat the study once the children reach the age of 15-16 years. Intervention studies among adolescents with psychiatric diagnoses may further illuminate the possible positive effect of a healthy eating pattern on mental health status.

\section{Conclusion}

We have shown a significant association between overall eating patterns and mental problems among young Norwegian adolescents (12-13 years of age), independent of physical activity, sedentary activity and background characteristics. Our findings support the proposition that a diverse, nutrient-dense diet and regular meals are associated with better mental health in adolescents, while energy-dense, nutrient-poor diets and irregular meals are associated with poorer mental health. Follow-up is needed to investigate the possible relationship between eating patterns and mental health among adolescents further.

\section{Acknowledgements}

Sources of funding: This research was supported by Telemark University College, Telemark Hospital, the Research Council of Norway, and the Public Health Programme for Telemark. Telemark University College, Telemark Hospital, the Norwegian Research Council and Telemark County Council had no role in the design, analysis or writing of this article. Conflicts of interest: The authors declare no conflict of interest. Authors' contributions: I.M.O. and M.V.S. were responsible for the study design and data collection. I.H. was responsible for the evaluation and choice of the SDQ as the instrument for mental health problem assessments. M.V.S. was responsible for the statistical analyses. All authors contributed to the interpretation of the results. I.M.O. drafted the manuscript and the other authors revised it critically. All authors read and approved the final version of the manuscript. Acknowledgements: The authors appreciate the cooperation of the children and their parents, the faculty staff of Telemark County primary and lower secondary schools, and the public health nurses who participated in this study.

\section{References}

1. Belfer ML (2008) Child and adolescent mental disorders: the magnitude of the problem across the globe. $J$ Child Psychol Psychiatry 49, 226-236.

2. Merikangas KR, He JP, Burstein M et al. (2010) Lifetime prevalence of mental disorders in US adolescents: results from the National Comorbidity Survey Replication Adolescent Supplement (NCS-A). J Am Acad Child Adolesc Psychiatry 49, 980-989.

3. Kessler RC, Berglund P, Demler O et al. (2005) Lifetime prevalence and age-of-onset distributions of DSM-IV disorders in the National Comorbidity Survey Replication. Arch Gen Psychiatry 62, 593-602.

4. Norwegian Institute of Public Health (2012) Mental Disorders in Children and Adolescents in Norway. Fact Sheet. Oslo: Norwegian Institute of Public Health.

5. Samdal O, Leversen I, Torsheim T et al. (2009) The Health Behaviour in School-Aged Children: WHO Collaborative Cross-National Study (HBSC). Bergen: Research Centre for Health Promotion, University of Bergen (in Norwegian).

6. Øverby N \& Andersen LF (2002) Ungkost 2000 (National Dietary Study among Students in 4th and 8th Grade). Oslo: Directorate of Health and Social Affairs (in Norwegian).

7. Bellisle F (2004) Effects of diet on behaviour and cognition in children. Br J Nutr 92, Suppl. 2, S227-S232.

8. Isaacs E \& Oates J (2008) Nutrition and cognition: assessing cognitive abilities in children and young people. Eur J Nutr 47, 4-24.

9. Øverby N \& Høigaard RNA (2012) Diet and behavioral problems at school in Norwegian adolescents. Food Nutr Res 2012, 56.

10. Weng TT, Hao JH, Qian QW et al. (2012) Is there any relationship between dietary patterns and depression and 
anxiety in Chinese adolescents? Public Health Nutr 15 , 673-682.

11. Azadbakht L \& Esmaillzadeh A (2012) Dietary patterns and attention deficit hyperactivity disorder among Iranian children. Nutrition 28, 242-249.

12. Lien L, Lien N, Heyerdahl S et al. (2006) Consumption of soft drinks and hyperactivity, mental distress, and conduct problems among adolescents in Oslo, Norway. Am J Public Health 10, 1815-1820.

13. Lien L (2007) Is breakfast consumption related to mental distress and academic performance in adolescents? Public Health Nutr 10, 422-428.

14. Robinson M, Kendall GE, Jacoby P et al. (2010) Lifestyle and demographic correlates of poor mental health in early adolescence. J Paediatr Child Health 47, 54-61.

15. Jacka FN, Kremer PJ, Berk M et al. (2011) A prospective study of diet quality and mental health in adolescents. Plos One 6, e24805.

16. Howard AL, Robinson M, Smith GJ et al. (2011) ADHD is associated with a 'Western' dietary pattern in adolescents. $J$ Atten Disord 15, 403-411.

17. Jacka FN, Kremer PJ, Leslie ER et al. (2010) Associations between diet quality and depressed mood in adolescents: results from the Australian Healthy Neighbourhoods Study. Aust N Z J Psychiatry 44, 435-442.

18. Oddy WH, Robinson M, Ambrosini GL et al. (2009) The association between dietary patterns and mental health in early adolescence. Prev Med 49, 39-44.

19. Wiles NJ, Northstone K, Emmett P et al. (2009) 'Junk food' diet and childhood behavioural problems: results from the ALSPAC cohort. Eur J Clin Nutr 63, 491-498.

20. O'Sullivan TA, Robinson M, Kendall GE et al. (2009) A good-quality breakfast is associated with better mental health in adolescence. Public Health Nutr 12, 249-258.

21. Jacques PF \& Tucker KL (2001) Are dietary patterns useful for understanding the role of diet in chronic disease? $A m J$ Clin Nutr 73, 1-2.

22. Hu FB (2002) Dietary pattern analysis: a new direction in nutritional epidemiology. Curr Opin Lipidol 13, 3-9.

23. Bamber DJ, Stokes CS \& Stephen AM (2007) The role of diet in the prevention and management of adolescent depression. Nutr Bull 32, 90-99.

24. Oellingrath I, Svendsen M \& Brantsaeter AL (2011) Tracking of eating patterns and overweight - a follow-up study of Norwegian school children from middle childhood to early adolescence. Nutr J 10, 106.

25. Goodman R (1999) The extended version of the Strengths and Difficulties Questionnaire as a guide to child psychiatric caseness and consequent burden. J Child Psychol Psychiatry 40, 791-799.

26. Oellingrath IM, Svendsen MV \& Brantsater AL (2010) Eating patterns and overweight in 9- to 10-year-old children in Telemark County, Norway: a cross-sectional study. Eur J Clin Nutr 64, 1272-1279.

27. Lillegaard IT, Øverby NC \& Andersen LF (2012) Evaluation of a short food frequency questionnaire used among children and adolescents. Food Nutr Res 56, 6399.

28. Schelling AB \& Streitlien A (2007) Utprøving av spørreskjema Kostholds- og maltidsmonster, fysisk aktivitet og vektutvikling (Pilot Study of the Questionnaire 'Diet and Eating Patterns, Physical Activity and Weight Development'). Notodden: Telemark Educational Research (in Norwegian).

29. Goodman R, Renfrew D \& Mullick M (2000) Predicting type of psychiatric disorder from Strengths and Difficulties Questionnaire (SDQ) scores in child mental health clinics in London and Dhaka. Eur Child Adolesc Psychiatry 9, 129-134.

30. Goodman R, Ford T, Simmons H et al. (2000) Using the Strengths and Difficulties Questionnaire (SDQ) to screen for child psychiatric disorders in a community sample. $\mathrm{BrJ}$ Psychiatry 177, 534-539.
31. Janz KF, Broffitt B \& Levy SM (2005) Validation evidence for the Netherlands physical activity questionnaire for young children: the Iowa bone development study. Res $Q$ Exerc Sport 76, 363-369.

32. Cole TJ, Flegal KM, Nicholls D et al. (2007) Body mass index cut offs to define thinness in children and adolescents: international survey. BMJ 335, 194.

33. Cole TJ, Bellizzi MC, Flegal KM et al. (2000) Establishing a standard definition for child overweight and obesity worldwide: international survey. BMJ 320, 1240-1243.

34. Van Roy B, Groholt B, Heyerdahl S et al. (2010) Understanding discrepancies in parent-child reporting of emotional and behavioural problems: effects of relational and socio-demographic factors. BMC Psychiatry 10, 56.

35. Heiervang E, Goodman A \& Goodman R (2008) The Nordic advantage in child mental health: separating health differences from reporting style in a cross-cultural comparison of psychopathology. J Child Psychol Psychiatry 49, 678-685.

36. Smith AP (2005) The concept of well-being: relevance to nutrition research. Br J Nutr 93, Suppl. 1, S1-S5.

37. Neumark-Sztainer D, Larson NI, Fulkerson JA et al. (2010) Family meals and adolescents: what have we learned from Project EAT (Eating Among Teens)? Public Health Nutr 13, $1113-1121$.

38. Ambrosini GL, Oddy WH, Robinson M et al. (2009) Adolescent dietary patterns are associated with lifestyle and family psycho-social factors. Public Health Nutr 12, $1807-1815$.

39. Cortese S, Isnard P, Frelut ML et al. (2007) Association between symptoms of attention-deficit/hyperactivity disorder and bulimic behaviors in a clinical sample of severely obese adolescents. Int J Obes (Lond) 31, 340-346.

40. Davis C (2010) Attention-deficit/hyperactivity disorder: associations with overeating and obesity. Curr Psychiatry Rep 12, 389-395.

41. Erhart M, Herpertz-Dahlmann B, Wille N et al. (2012) Examining the relationship between attention-deficit/ hyperactivity disorder and overweight in children and adolescents. Eur Child Adolesc Psychiatry 21, 39-49.

42. Bitsakou P, Psychogiou L, Thompson M et al. (2009) Delay aversion in attention deficit/hyperactivity disorder: an empirical investigation of the broader phenotype. Neuropsychologia 47, 446-456.

43. Gibson EL (2012) The psychobiology of comfort eating: implications for neuropharmacological interventions. Behav Pharmacol 23, 442-460.

44. Van Roy B, Groholt B, Heyerdahl S et al. (2006) Selfreported strengths and difficulties in a large Norwegian population 10-19 years: age and gender specific results of the extended SDQ-questionnaire. Eur Child Adolesc Psychiatry 15, 189-198.

45. Achenbach TM, Becker A, Dopfner M et al. (2008) Multicultural assessment of child and adolescent psychopathology with ASEBA and SDQ instruments: research findings, applications, and future directions. J Child Psychol Psychiatry 49, 251-275.

46. Obel C, Heiervang E, Rodriguez A et al. (2004) The Strengths and Difficulties Questionnaire in the Nordic countries. Eur Child Adolesc Psychiatry 13, Suppl. 2, II32-II39.

47. Bourdon KH, Goodman R, Rae DS et al. (2005) The strengths and difficulties questionnaire: US normative data and psychometric properties. J Am Acad Child Adolesc Psychiatry 44, 557-564.

48. Ravens-Sieberer U, Wille N, Erhart M et al. (2008) Prevalence of mental health problems among children and adolescents in Germany: results of the BELLA study within the National Health Interview and Examination Survey. Eur Child Adolesc Psychiatry 17, 22-33. 
49. Goodman R (2001) Psychometric properties of the strengths and difficulties questionnaire. J Am Acad Child Adolesc Psychiatry 40, 1337-1345.

50. Livingstone MB \& Robson PJ (2000) Measurement of dietary intake in children. Proc Nutr Soc 59, 279-293.

51. Drewnowski A (2001) Diet image: a new perspective on the food-frequency questionnaire. Nutr Rev 59, 370-372.

52. Newby PK (2007) Are dietary intakes and eating behaviors related to childhood obesity? A comprehensive review of the evidence. J Law Med Ethics 35, 35-60.

53. Togo P, Heitmann BL, Sorensen TIA et al. (2003) Consistency of food intake factors by different dietary assessment methods and population groups. BrJ Nutr 90, 667-678.

54. Khani BR, Ye W, Terry P et al. (2004) Reproducibility and validity of major dietary patterns among Swedish women assessed with a food-frequency questionnaire. J Nutr $\mathbf{1 3 4}$ $1541-1545$.

55. Hu FB, Rimm E, Smith-Warner SA et al. (1990) Reproducibilty and validity of dietary patterns assessed with a food-frequency questionnaire. Am J Clin Nutr 69, 243-249.

56. Ambrosini GL, O'Sullivan TA, de Klerk NH et al. (2011) Relative validity of adolescent dietary patterns: a comparison of a FFQ and $3 \mathrm{~d}$ food record. Br J Nutr 105, 625-633.

57. Page AS, Cooper AR, Griew P et al. (2010) Viewing is related to psychological difficulties irrespective of physical activity. Pediatrics 126, E1011-E1017.

58. Bremnes AJ, Martiniussen M, Laholt $\mathrm{H}$ et al. (2011) The association between mental health and physical activity among high-school students. J Norw Psychol Assess 48, 332-338. 